\title{
EDITORIAL
}

\section{The functional architecture of the prefrontal cortex and schizophrenia ${ }^{1}$}

Findings from a number of studies have implicated the prefrontal cortex (PFC), especially the dorsolateral region, as a site of dysfunction in schizophrenia (Goldberg et al. 1989). For example, in contrast to controls, schizophrenics fail to activate the dorsolateral PFC while engaged in the Wisconsin Card Sort Task (Weinberger et al. 1986), and they exhibit an inability to learn how to perform this task (Goldberg et al. 1987). Schizophrenic subjects also demonstrate significant impairments on oculomotor delayed-response tasks (Park \& Holzman, 1992), a behavioural paradigm that in nonhuman primates appears to be a selective and reliable measure of dorsolateral PFC function (Fuster, 1989; Funahashi et al. 1991, 1993). Despite these clear deficits in PFC functioning in schizophrenia, the apparent lack of gross structural abnormalities in the PFC of schizophrenic subjects suggests that this dysfunction may be a consequence of more subtle alterations in the intrinsic neural circuitry of this region. Understanding how such disturbances in connectivity might occur in schizophrenia requires knowledge of the normal functional architecture of the PFC. In addition, the PFC is functionally coupled with multiple other brain regions, some of which may also be impaired in schizophrenia. Consequently, it is important to understand how information processing within the PFC influences, and is influenced by, the activity of these regions.

Any hypothesis of PFC dysfunction in schizophrenia also needs to account for the typical onset of the symptoms of this disorder, including impairments in cognitive processes (Heaton et al. 1994), during late adolescence or young adulthood (Carpenter \& Buchanan, 1994). In addition, hypotheses of PFC dysfunction in schizophrenia should consider the possible role of dopamine, which has long been considered to play a part in the pathophysiology of this disorder. Although our knowledge of these factors remains limited in many respects, recent advances in understanding the normal functional architecture of the primate PFC, in concert with post-mortem studies of schizophrenic patients, have provided a basis for the generation of novel, testable hypotheses regarding the pathophysiology of PFC dysfunction in this illness.

\section{FUNCTIONAL ARCHITECTURE OF THE PRIMATE PREFRONTAL CORTEX}

Many of the PFC-mediated tasks on which schizophrenic subjects perform poorly share as a common feature the involvement of working memory (Goldman-Rakic, 1987) or the temporal integration of information (Fuster, 1985); that is, the experimental subject has to retain knowledge of the information provided by an environmental cue in order to perform the appropriate behavioural response after the cue has been removed. In spatial delayed-response tasks in monkeys, animals are required to hold 'on line', or in working memory, the location of an environmental cue in space during a delay period following the removal of the cue. By recording from micro-electrodes in the dorsolateral PFC of monkeys as they perform such tasks, it has been possible to correlate neuronal activity with cognitive processes. Separate populations of PFC neurons have been identified whose activity is temporally linked to specific components of the delayed-response task

\footnotetext{
1 Address for correspondence: Dr David A. Lewis, University of Pittsburgh, Western Psychiatric Institute and Clinic, 3811 O'Hara Street, BST W1651, Pittsburgh, PA 15213, USA.
} 
(Fuster et al. 1982). The firing rate of one neuronal population appears to be associated with maintaining the representation of the environmental cue, whereas the firing rate of a second population of neurons appears to be associated with the preparation for the motor response (Quintana \& Fuster, 1992). Although the available data are limited, these neuronal populations appear to be present in clusters that are widely distributed across regions of the PFC (Fuster, 1993). Cooling the dorsolateral PFC, which impairs the ability of both neuronal populations to maintain an elevated firing rate, also impairs the animals' performance on the task (Bauer \& Fuster, 1976). In addition, in individual delayed-response trials, failure to perform the appropriate response has been found to parallel the inability of individual neurons to maintain an elevated firing rate during the cue-response interval (Funahashi et al. 1989).

The discovery of groups of neurons in the dorsolateral PFC, which seem to be involved in the temporary memorization of the meaning of a sensory cue, and in the preparation for a learned behavioural response, has led to the refinement of models of PFC functioning. While differences in some specific aspects of these models exist among researchers, they share the temporally coordinated activation of spatially segregated, but highly interconnected networks of PFC neurons, with each group of neurons attending to specific aspects of the task at hand.

One possible mechanism for the coordinated recruitment of distributed networks of PFC neurons may be the specific patterns of intrinsic connections provided by the axon collaterals of pyramidal neurons. These cells, the major class of excitatory cortical neurons (Feldman, 1984), are characterized by the presence of an apical dendrite, dendritic spines and an axon that projects to other brain regions. Their main axonal trunk also gives rise to recurrent axon collaterals, which appear to synapse predominantly with dendritic spines (LeVay, 1988; McGuire et al. 1991), indicating that their main targets are other pyramidal neurons. Thus, these collaterals serve as the major propagators of intrinsic, or intra-areal, excitatory activity in the primate cerebral cortex. In sensory and motor regions, some axon collaterals extend for considerable distances horizontally and form clusters of terminal fields that are distributed as patches (Rockland \& Lund, 1983; Livingstone \& Hubel, 1984; DeFelipe et al. 1986; Matsubara et al. 1987; King et al. 1989; Juliano et al. 1990). These clustered horizontal connections appear to link columns of cells with preferences for specific types of stimuli (T'so et al. 1986; Matsubara \& Phillips, 1988; T'so \& Gilbert, 1988; Wallace et al. 1991). In monkey PFC, the axon collaterals of a subpopulation of pyramidal neurons also extend for considerable distances horizontally. However, these intrinsic axon collaterals give rise to clusters of axon terminals in the superficial layers (see Fig. 1) which form a distinctive lattice-like structure organized as a series of stripes (Levitt et al. 1993). Although the results of direct physiological investigations of these lattice connections are not yet available, by analogy to sensory regions, this lattice structure of interconnected stripes may link the activity of spatially segregated populations of PFC neurons. For example, combined retrograde and anterograde tracing studies have demonstrated that reciprocal divergent and convergent projections are present among lattice stripes of monkey PFC (Pucak et al. 1994). These findings raise the possibility that intrinsic lattice connections could be the substrate for a reverberating cortical circuit that maintains and coordinates the activity of specific populations of PFC neurons during the delay phase of delayedresponse tasks. Pyramidal neurons in layer 3 are major contributors of the axon collaterals that form the intrinsic lattice, and consequently these neurons may play a particularly vital role in the integrated activity of specific groups of PFC neurons. It is interesting in this regard that when monkeys were engaged in a spatial delayed-response task, glucose utilization was found to be greatest in layer 3 of the dorsolateral PFC (Friedman \& Goldman-Rakic, 1994).

In addition to pyramidal neurons in layer 3, pyramidal cells in layers 2 and 5 contribute axon collaterals to the lattice structure of intrinsic excitatory connections (Levitt et al. 1993). The main axonal trunks of some pyramidal neurons in layers 2, 3 and 5 enter the white matter and provide the principal source of PFC connections to other cortical regions and some subcortical structures such as the striatum. Thus, the axons of these neurons are equipped not only to participate in the coordination of activity among groups of PFC neurons, but also to convey that information to more broadly distributed networks of neurons. Indeed, recent studies have shown that individual 


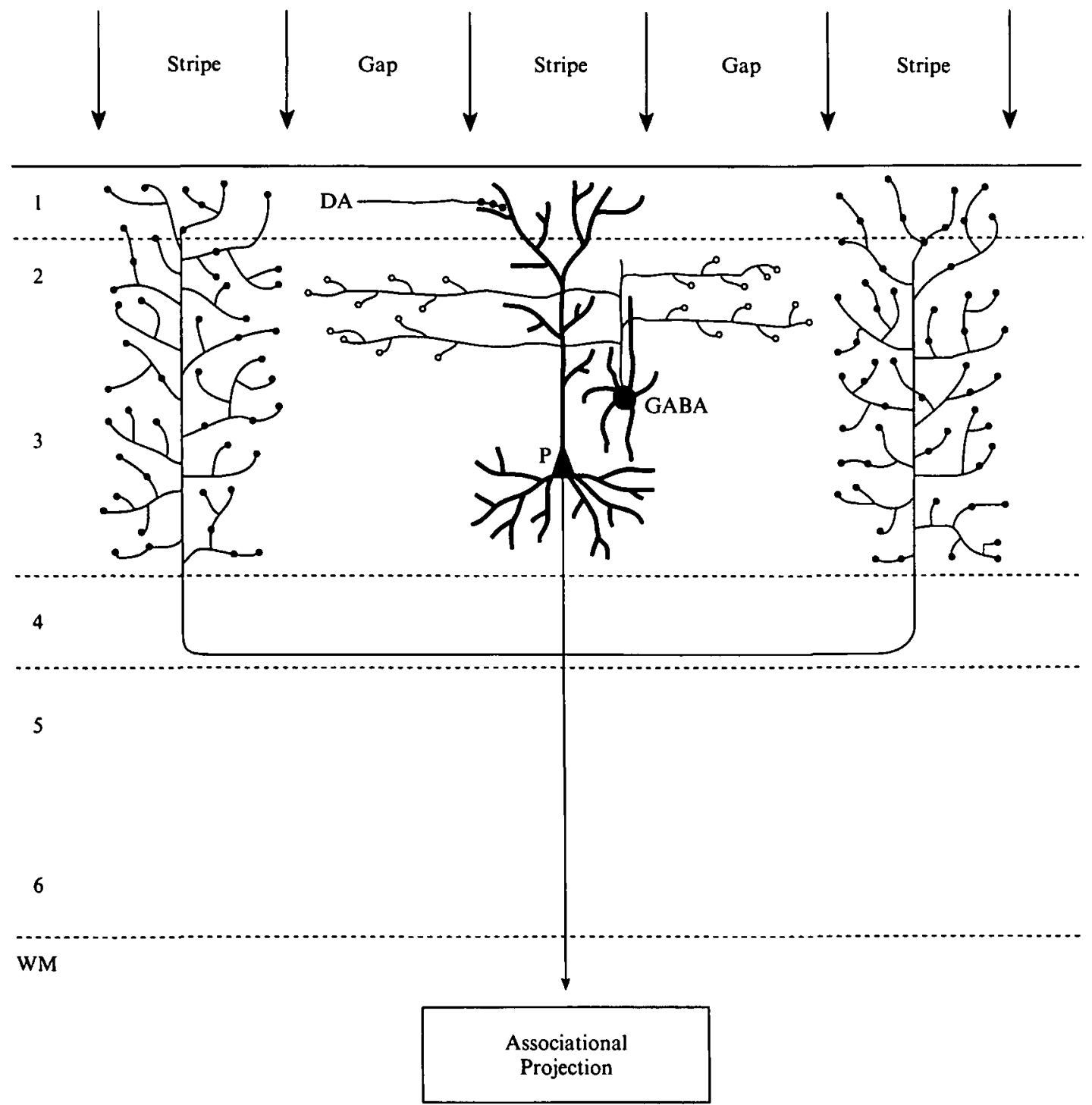

FIG. 1. Hypothetical model of components of the intrinsic circuitry of primate prefrontal cortex. A subpopulation of pyramidal neurons $(P)$ in layer 3 furnishes associational projections to other cortical regions, as well as collaterals that spread horizontally and arborize in discrete clusters arrayed as stripes (intrinsic lattice). The activity of these neurons may be modulated by dopamine (DA) afferents, in addition to other inputs. Zones of inhibitory activity in the gaps between the stripes of the intrinsic lattice may be furnished by a particular subpopulation of GABA neurons, the wide arbor neurons. See text for additional details.

PFC pyramidal neurons provide both intrinsic axon collaterals to lattice stripes and axonal projections to other cortical regions (Melchitzky et al. 1994).

Thus, the patterns of intra- and inter-areal connectivity subserved by these populations of PFC neurons suggest that they may be critical components of the neural circuitry underlying the distinctive behavioural tasks mediated by the PFC. Consequently, these connections must be strongly considered as possible suspects for the disrupted or altered circuitry that is manifest as PFC dysfunction in schizophrenia. 


\section{DEVELOPMENT OF PFC CIRCUITRY AND THE ONSET OF SCHIZOPHRENIC SYMPTOMS}

A critical test of any explanation of PFC dysfunction in schizophrenia is its ability to account for the typical onset of the symptoms of this disorder, including cognitive impairment (Heaton et al. 1994), during late adolescence and early adulthood. Studies in both monkeys and humans have demonstrated that the period of adolescence is associated with a marked reduction in the density of PFC synapses (Huttenlocher, 1979; Bourgeois et al. 1994). In monkeys, the greatest degree of change appears to involve asymmetric (excitatory) axospinous synapses in the supragranular layers (Bourgeois et al. 1994). Consistent with these observations, the density of dendritic spines on layer 3 pyramidal neurons in monkey PFC has been observed to decline by $50 \%$ between the ages of 2 and 4 years (Anderson et al. 1995), the period of adolescence in this species (Plant, 1988). Because dendritic spines are the primary site of excitatory inputs to these neurons (Colonnier, 1968; LeVay, 1973; Mates \& Lund, 1983), these findings suggest that layer 3 pyramidal neurons lose a substantial portion of their excitatory input during the late functional maturation of the PFC. Given that the majority of cortical synapses are derived from intrinsic sources (White, 1989), the synapses formed by axon collaterals of pyramidal neurons are likely candidates for this loss. In contrast, the other major source of excitatory inputs to the superficial cortical layers, associational and callosal corticortical afferents, would have to be pruned to a very high extent to produce a total decrease of $50 \%$ in synaptic density. In addition, the number of axons in the corpus callosum of monkeys decreases from a peak at birth to reach stable levels by 6 months of age (LaMantia \& Rakic, 1990), suggesting that these axons may be less likely to be involved in the peripubertal attrition of PFC excitatory synapses. Consequently, the loss during the peripubertal period of a large proportion of synapses furnished by axon collaterals of lattice neurons could refine the pattern of discontinuities in the intrinsic connections furnished by each pyramidal neuron, and give rise, during late adolescence, to the mature form of the intrinsic lattice structure.

Disturbances in this developmental process could produce abnormalities in intrinsic PFC circuitry that might underlie the PFC dysfunction present in schizophrenia (Feinberg, 1982; Hoffman \& Dobscha, 1989). For example, if the speculations discussed above about the role of lattice connectivity in PFC function are correct, then a deficient number of lattice connections could impair the sustained activity of specific networks of PFC neurons required for tasks involving working memory (Goldman-Rakic, 1987) or the integration of cross-temporal contingencies (Fuster, 1985). Thus, abnormalities in lattice connections might produce the types of PFC dysfunction that are characteristic of schizophrenia. Alternatively, the maturation of the intrinsic lattice structure might unmask the functional consequences of abnormalities in PFC circuitry that occurred earlier in development (Weinberger, 1987; Benes et al. 1991, 1992; Akbarian et al. 1993).

Hypotheses of PFC dysfunction in schizophrenia should also consider the possible role of dopaminergic (DA) systems in this disorder. Although the data are quite limited, both clinical studies (Doran et al. 1987; Weinberger et al. 1988; Breier et al. 1993) and theoretical modelling (Cohen \& Servan-Schreiber, 1992) have suggested that PFC dysfunction in schizophrenia may be associated with abnormalities in the DA innervation of this region. In addition, animal studies have clearly demonstrated that an intact DA innervation of PFC is critical for the functional integrity of this region on delayed-response tasks (Brozoski et al. 1979; Sawaguchi \& Goldman-Rakic, 1991; Roberts et al. 1994). Interestingly, the available data indicate that DA afferents to PFC innervate pyramidal neurons (Goldman-Rakic et al. 1989; Sesack et al. 1995; Smiley \& Goldman-Rakic, 1993), at least some of which may participate in lattice connectivity (see Fig. 1). In addition, some dendritic spines receive convergent input from a Type 1 (asymmetric) excitatory synapse and a Type 2 (symmetric) DA synapse (Goldman-Rakic et al. 1989). Although the source of these asymmetric synapses has not been determined, this arrangement raises the possibility that DA afferents may directly modulate, at the level of dendritic spines, activity within the intrinsic lattice structure of excitatory connections.

In addition, the DA innervation of PFC also undergoes substantial changes during adolescence 
that may be temporally linked to the developmental refinements in lattice connectivity described above. For example, the density of DA varicosities in layer 3 of monkey dorsolateral PFC reaches a peak in animals 2-3 years-of-age, before declining to stable adult levels (Rosenberg \& Lewis, 1995). The possible relationship of these changes to the maturation of lattice connectivity is further strengthened by the fact that the developmental changes in DA innervation appear to be specific to layer 3 in that such developmental changes do not occur in the more superficial or deep cortical layers (Rosenberg \& Lewis, 1995). Consequently, alterations in the intrinsic lattice structure of PFC connectivity in schizophrenia may be reflected in altered DA function of this region.

\section{IMPLICATIONS FOR PFC DYSFUNCTION IN SCHIZOPHRENIA}

As suggested above, the typical late adolescent/early adult onset of schizophrenia may represent the behavioural manifestation of an abnormality in the maturation of the intrinsic lattice organization during this period of development. Indeed, several lines of evidence from studies of the brains of schizophrenic subjects are consistent with the hypothesis that schizophrenia is characterized by a decrease in the intrinsic connectivity of dorsolateral PFC. For example, although the total number of cortical neurons does not appear to be altered in schizophrenia (Pakkenberg, 1993; Akbarian et al. 1995), cell packing density is increased in the PFC in schizophrenic subjects (Pakkenberg, 1993; Selemon et al. 1993; Daviss \& Lewis, 1995), suggesting that the amount of cortical neuropil is decreased. In addition, a decreased density of dendritic spines on layer 3 pyramidal neurons (Garey et al. 1994), lower measures of synaptic markers (Glantz \& Lewis, 1994) and loss of synaptic building blocks (Pettegrew et al. 1991) have recently been reported in the PFC of schizophrenic subjects. These observations could all be attributable to a common process that reduced the number of intrinsic connections, which account for over two-thirds of all cortical synapses (White, 1989). In addition, at least some recent findings with brain imaging techniques, such as decreased PFC activation as measured by PET (Buchsbaum et al. 1992) and decreased cortical grey matter thickness as measured by MRI (Zipursky et al. 1992; Schlaepfer et al. 1994; Seidman et al. 1994), may also be consistent with a defect in PFC connectivity in schizophrenic subjects. In these imaging studies, and those involving MRI spectroscopy (Pettegrew et al. 1991), the reported differences between schizophrenics and controls are in the same direction as those changes found to occur normally over the adolescent age range (for review, see Keshavan et al. 1994).

How might the failure to develop the normal lattice organization of intrinsic connections in the PFC result in the symptoms of schizophrenia? One possibility is that, without an intact lattice structure, the recruitment of the networks of neurons necessary for the temporal organization of thought processes is impaired. For example, disrupted lattice connections could limit the coordinated activity of the components of the neural network responsible for holding 'on line' a specific set of stimulus characteristics. Moreover, such a disturbance of connectivity could also impair the flow of information between the groups of neurons responsible for remembering these characteristics, and those involved in preparing an appropriate response. The failure to maintain properly the relevant bits of information in working memory and the subsequent impairment of the preparation of an appropriate response might be reflected in a disorder of the temporal organization of thought and language, producing symptoms such as loose associations (Fuster, 1993). Finally, because of its role in the temporal organization of thought processes that involve other cortical regions, a deficit in the intrinsic connectivity of the PFC may also contribute to the production of symptoms that have been generally related to the dysfunction of these regions.

This view posits that the dysfunction of the PFC is due to a 'fixed' lesion of intrinsic excitatory connections that does not vary with time after the mature pattern of those connections is achieved in early adulthood. However, it must be kept in mind that the severity of the symptoms attributed to this deficit could vary with changes in other dynamic aspects of PFC circuitry. For example, one class of GABAergic local circuit neuron in monkey PFC, the wide arbor neuron (Lund \& Lewis, 1993), has an axon arbor that may be specialized for establishing zones of inhibition between the 
stripes of the intrinsic lattice structure (Levitt et al. 1993; see Fig. 1). Activity-dependent changes in the functional properties of these inhibitory neurons (Jones, 1993) could have, in turn, an impact on the fidelity of information processing in the remaining lattice connections by either sharpening or distorting the boundaries of the interconnected lattice stripes. In this regard, it is interesting to note that several recent studies of local circuit neurons in the PFC of schizophrenic subjects have revealed the altered expression of functionally important proteins in GABAergic neurons, in the absence of any apparent changes in the number of these neurons (Akbarian et al. 1995; Daviss \& Lewis, 1995).

\section{CONCLUSION}

The recent increases in knowledge about the functional architecture of the primate PFC, combined with imaging and post-mortem studies of the brains of schizophrenic subjects, make it possible to generate new hypotheses regarding the neurobiological substrates of PFC dysfunction in this illness. In this essay we suggest the hypothesis that PFC dysfunction in schizophrenia results from alterations in the intrinsic lattice structure of excitatory connectivity in the PFC. However, significant gaps in our understanding remain. For example, what are the physiological properties of lattice connections in the PFC? What is the precise time course of the functional maturation of the lattice structure and what factors are important in the regulation of its development? What are the anatomical and functional relationships between lattice connections and dopaminergic afferents to the PFC? How accurately do studies in non-human primates predict the organization of the intrinsic circuitry of human PFC? What role do lattice connections actually play in the temporal organization of thought processes? The integration of basic and clinical studies of the functional architecture and development of the PFC may provide answers to these questions, as well as clearer insights into the mechanisms that underlie PFC dysfunction in schizophrenia.

DAVID A. LEWIS AND STEWART A. ANDERSON

Studies by the authors cited in this manuscript were supported by USPHS grants MH45156 and MH43784, and Research Scientist Development Award MH00519.

\section{REFERENCES}

Akbarian, S., Bunney, W. E., Jr., Potkin, S. G., Wigal, S. B., Hagman, J. O., Sandman, C. A. \& Jones, E. G. (1992). Altered distribution of nicotinamide-adenine dinucleotide phosphatediaphorase cells in frontal lobe of schizophrenics implies disturbances of cortical development. Archives of General Psychiatry 50, 169-177.

Akbarian, S., Kim, J. J., Potkin, S. G., Hagman, J. O., Tafazzoli, A., Bunney, W. E., Jr. \& Jones, E. G. (1995). Gene expression for glutamic acid decarboxylase is reduced without loss of neurons in prefrontal cortex of schizophrenics. Archives of General Psychiatry 52, 258-266.

Anderson, S. A., Classey, J. D., Condé, F., Lund, J. S. \& Lewis, D. A. (1995). Postnatal development of excitatory and inhibitory inputs to layer III pyramidal cells in monkey prefrontal cortex. Neuroscience 67, 7-22.

Bauer, R. H. \& Fuster, J. M. (1976). Delayed matching and delayedresponse deficit from cooling dorsolateral prefrontal cortex in monkeys. Journal of Comparative Physiology and Psychology 90 , 293-302.

Benes, F. M., McSparren, J., Bird, E. D., San Giovani, J. P. \& Vincent, S. L. (1991). Deficits in small interneurons in prefrontal and cingulate cortices of schizophrenic and schizoaffective patients. Archives of General Psychiary 48, 996-1001.

Benes, F. M., Vincent, S. L., Alsterberg, G., Bird, E. D. \& San Giovanni, J. P. (1992). Increased GABAa receptor binding in superficial layers of cingulate cortex in schizophrenics. Journal of Neuroscience 12, 924-929.
Bourgeois, J.-P., Goldman-Rakic, P. S. \& Rakic, P. (1994). Synaptogenesis in the prefrontal cortex of rhesus monkeys. Cerebral Cortex 4, 78-96.

Breier, A., David, O. R., Buchanan, R. W., Moricle, L. A. \& Munson, R. C. (1993). Effects of metabolic perturbation of plasma homovanillic acid in schizophrenia: relationship to prefrontal cortical volume. Archives of General Psychiatry 50, 541-550.

Brozoski, T.J., Brown, R. M., Rosvold, H. E. \& Goldman, P. S. (1979). Cognitive deficit caused by regional depletion of dopamine in prefrontal cortex of rhesus monkeys. Science 205, 929-932.

Buchsbaum, M. S., Haier, R. J., Potkin, S. G., Neuchterlein, K., Bracha, H. S., Katz, M., Lohr, J., Wu, J., Lottenberg, S., Jerabek, P. A., Trenary, M., Tafalla, R., Reynolds, C. \& Bunney, W. E., Jr. (1992). Frontrostriatal disorder of cerebral metabolism in nevermedicated schizophrenics. Archives of General Psychiarry 49, 935-942.

Carpenter, W. T. Jr. \& Buchanan, R. W. (1994). Schizophrenia. New England Journal of Medicine 330, 681-690.

Cohen, J. D. \& Servan-Schreiber, D. (1992). Context, cortex, and dopamine: a connectionist approach to behavior and biology in schizophrenia. Psychological Review 99, 45-77.

Colonnier, M. (1968). Synaptic patterns on different cell types in the different laminae of the cat visual cortex. An electron microscope study. Brain Research 9, 268-287.

Daviss, S. R. \& Lewis, D. A. (1995). Local circuit neurons of the prefrontal cortex in schizophrenia: selective increase in the density of calbindin-immunoreactive neurons. Psychiatry Research (in the press).

DeFelipe, J., Conley, M. \& Jones, E. G. (1986). Long range local 
collateralisation of axons arising from corticocortical cells in monkey sensory motor cortex. Journal of Neuroscience 6, 37493766 .

Doran, A. R., Boronow, J., Weinberger, D. R., Wolkowitz, O. M., Breier, A. \& Pickar, D. (1987). Structural brain pathology in schizophrenia revisited: prefrontal cortex pathology is inversely correlated with cerebrospinal fluid levels of homovanillic acid. Neuropsychopharmacology 1, 25-32.

Feinberg, 1. (1982). Schizophrenia: caused by a fault in programmed synaptic elimination during adolescence? Journal of Psychiatric Research 17, 319-334.

Feldman, M. L. (1984). Morphology of the neocortical pyramidal neuron. In Cerebral Cortex. Cellular Components of the Cerebral Cortex (ed. A. Peters and E. G. Jones), pp. 123-200. Plenum Press: New York.

Friedman, H. R. \& Goldman-Rakic, P. S. (1994). Coactivation of prefrontal cortex and inferior parietal cortex in working memory tasks revealed by $2 \mathrm{DG}$ functional mapping in the rhesus monkey. Journal of Neuroscience 14, 2775-2788.

Funahashi, S., Bruce, C. J. \& Goldman-Rakic, P. S. (1989). Mnemonic coding of visual space in the monkey's dorsolateral prefrontal cortex. Journal of Neurophysiology 61, 331-349.

Funahashi, S., Bruce, C. J. \& Goldman-Rakic, P. S. (1991). Neuronal activity related to saccadic eye movements in the monkey's dorsolateral prefrontal cortex. Journal of Neurophysiology 65, $1464-1483$.

Funahashi, S., Bruce, C. J. \& Goldman-Rakic, P. S. (1993). Dorsolateral prefrontal lesions and oculomotor delayed-response performance: evidence for mnemonic 'scotomas'. Journal of Neuroscience 13, 1479-1497.

Fuster, J. M. (1985). The prefrontal cortex, mediator of crosstemporal contingencies. Human Neurobiology 4, 169-179.

Fuster, J. M. (1989). The Prefrontal Cortex: Anatony, Physiology and Neuropsychology of the Frontal Lobe. Raven Press: New York.

Fuster, J. M. (1993). Frontal lobes. Current Opinion in Neurobiology $3,160-165$.

Fuster, J. M., Bauer, R. H. \& Jervey, J. P. (1982). Cellular discharge in the dorsolateral prefrontal cortex of the monkey in cognitive tasks. Experimental Neurology 77, 679-694.

Garey, L. J., Patel, T. \& Ong, W. Y. (1994). Loss of dendritic spines from cortical pyramidal cells in schizophrenia. Schizophrenia Research 11, 137.

Glantz, L. A. \& Lewis, D. A. Synaptophysin and not RABA3 is specifically reduced in the prefrontal cortex of schizophrenic subjects. Society for Neuroscience Abstracts 20, 622.

Goldberg, T. E., Weinberger, D. R., Berman, K. F., Pliskin, N. H. \& Podd, M. H. (1987). Further evidence for dementia of the prefrontal type in schizophrenia? Archives of General Psychiarry 44, 1008-1014.

Goldberg, T. E., Berman, K. F. \& Weinberger, D. R. (1989). An orientation to work on the prefrontal cortex in schizophrenia. In Schizophrenia: Scientific Progress (ed. S. C. Schultz and C. A. Tamminga), pp. 227-246. Oxford University Press: New York.

Goldman-Rakic, P. S. (1987). Circuitry of primate prefrontal cortex and regulation of behavior by representational memory. In Handbook of Physiology. The Nervous System, vol. 5 (ed. F. Plum and V. Mountcastle), pp. 373-417. American Physiological Society: Bethesda, MD.

Goldman-Rakic, P. S., Leranth, C., Williams, S. M., Mons, N. \& Geffard, M. (1989). Dopamine synaptic complex with pyramidal neurons in primate cerebral cortex. Proceedings of the National Academy of Sciences USA 86, 9015-9019.

Heaton, R., Paulsen, J. S., McAdams, L. A., Kuck, J., Zisook, S., Braff, D., Harris, J. \& Jeste, D. V. (1994). Neuropsychological deficits in schizophrenics: relationship to age, chronicity, and dementia. Archives of General Psychiatry 51, 469-476.

Hofiman, R. E. \& Dobscha, S. K. (1989). Cortical pruning and the development of schizophrenia: a computer model. Schizophrenia Bulletin 15, 477-490.

Huttenlocher, P. R. (1979). Synaptic density in human frontal cortex - developmental changes and effects of aging. Brain Research 163, 195-205.

Jones, E. G. (1993). GABAergic neurons and their role in cortical plasticity in primates. Cerebral Cortex 3, 361-372.

Juliano, S. L., Friedman, D. P. \& Eslin, D. E. (1990). Corticocortical connections predict patches of stimulus-evoked metabolic activity in monkey somatosensory cortex. Journal of Comparative Neurology 298, 23-39.

Keshavan, M. S., Anderson, S. \& Pettegrew, J. W. (1994). Is schizophrenia due to excessive synaptic pruning in the prefrontal cortex? The Feinberg hypothesis revisited. Journal of Psychiatric Research 28, 239-265.

King, M. A., Louis, P. M., Hunter, B. E. \& Walker, D. W. (1989). Biocytin: a versatile anterograde neuroanatomical tract-tracing alternative. Brain Research 497, 361-367.

LaMantia, A.-S. \& Rakic, P. (1990). Axon overproduction and elimination in the corpus callosum of the developing rhesus monkey. Journal of Neuroscience 10, 2156-2175.

LeVay, S. (1973). Synaptic patterns in the visual cortex of the cat and monkey. Electron microscopy of Golgi preparations. Journal of Comparative Neurology 150, 53-86.

LeVay, S. (1988). Patchy intrinsic projections in visual cortex, area 18, of the cat: morphological and immunocytochemical evidence for an excitatory function. Journal of Comparative Neurology 269, 265-275.

Levitt, J. B., Lewis, D. A., Yoshioka, T. \& Lund, J. S. (1993). Topography of pyramidal neuron intrinsic connections in macaque monkey prefrontal cortex (areas $9 \& 46$ ). Journal of Comparative Neurology 338, 360-376.

Livingstone, M. S. \& Hubel, D. H. (1984). Specificity of intrinsic connections in primate primary visual cortex. Journal of Neuroscience 4, 2830-2835.

Lund, J. S. \& Lewis, D. A. (1993). Local circuit neurons of developing and mature macaque prefrontal cortex: Golgi and immunocytochemical characteristics. Journal of Comparative Neurology 328, 282-312.

McGuire, B. A., Gilbert, C. D., Rivlin, P. K. \& Wiesel, T. N. (1991). Targets of horizontal connections in macaque primary visual cortex. Journal of Comparative Neurology 305, 370-392.

Mates, S. L. \& Lund, J. S. (1983). Spine formation and maturation of type 1 synapses on spiny stellate neurons in primate visual cortex. Journal of Comparative Neurology 221, 91-97.

Matsubara, J. A., Cynader, M.S. \& Swindale, N. V. (1987) Anatomical properties and physiological correlates of the intrinsic connections in cat area 18. Journal of Neuroscience 7, 1428-1446.

Matsubara, J. A. \& Phillips, D. P. (1988). Intracortical connections and their physiological correlates in the primary auditory cortex (AI) of the cat. Journal of Comparative Neurology 268, 38-48.

Melchitzky, D. S., Pucak, M. L., Dammerman, R. S. \& Lewis, D. A. (1994). Morphology and extrinsic targets of pyramidal neurons furnishing intra-areal connections in monkey prefrontal cortex. Society for Neuroscience Abstracts 20, 1416.

Pakkenberg, B. (1993). Total nerve cell number in neocortex in chronic schizophrenics and controls estimated using optical disectors. Biological Psychiatry 34, 768-772.

Park, S. \& Holztman, P. S. (1992). Schizophrenics show spatial working memory deficits. Archives of General Psychiarry 49, 975-982.

Pettegrew, J. W., Keshavan, M. S., Panchalingam, K., Strychor, S., Kaplan, D. B., Tretta, M. G. \& Allen, M. (1991). Alterations in brain high-energy phosphate and membrane phospholipid metabolism in first-episode, drug-naive schizophrenics. Archives of General Psychiatry 48, 563-568.

Plant, T. M. (1988). Neuroendocrine basis of puberty in the rhesus monkey (Macca mulatta). In Frontiers in Neuroendocrinology, vol. 10 (ed. L. Martin and W. F. Ganong), pp. 215-238. Raven Press: New York.

Pucak, M. L., Levitt, J. B., Classey, J. D., Lund, J. S. \& Lewis, D. A. (1994). Comparison of intra- and inter-areal patterns of connectivity in monkey prefrontal cortex. Society for Neuroscience Abstracts 20, 1416. 
Quintana, J. \& Fuster, J. (1992). Menemonic and predictive functions of cortical neurons in a memory task. Neuro Report 3, 721-724.

Roberts, A. C., DeSalvia, M. A., Wilkinson, L. S., Collins, P., Muir, J. L., Everitt, B. J. \& Robbins, T. W. (1994). 6-Hydroxydopamine lesions of the prefrontal cortex in monkeys enhance performance on an analog of the Wisconsin Card Sort Test: possible interactions with subcortical dopamine. Journal of Neuroscience 14, 2531-2544.

Rockland, K. S. \& Lund, J.S. (1983). Intrinsic laminar lattice connections in primate visual cortex. Journal of Comparative Neurology 216, 303-318.

Rosenberg, D. R. \& Lewis, D. A. (1995). Postnatal maturation of the dopaminergic innervation of monkey prefrontal and motor cortices: a tyrosine hydroxylase immunohistochemical analysis. Journal of Comparative Neurology 358, 383-400.

Sawaguchi, T. \& Goldman-Rakic, P. S. (1991). Dl dopamine receptors in prefrontal cortex: involvement in working memory. Science 251, 947-251.

Schlaepfer, T. E., Harris, G. J., Tien, A. Y., Peng, L. W., Lee, S., Federman, E. B., Chase, G. A., Barta, P. E. \& Pearlson, G. D. (1994). Decreased regional cortical gray matter volume in schizophrenia. American Journal of Psychiatry 151, 842-848.

Seidman, L. J., Yurgelun-Todd, D., Kremen, W. S., Woods, B. T., Goldstein, J. M., Faraone, S. V. \& Tsuang, M. T. (1994). Relationship of prefrontal and temporal lobe MRI measures to neuropsychological performance in chronic schizophrenia. Biological Psychiatry 35, 235-246.

Selemon, L. D., Rajkowska, G. \& Goldman-Rakic, P. S. (1993). Cytologic abnormalities in area 9 of the schizophrenic cortex. Society for Neuroscience Abstracts 19, 200.

Sesack, S. R., Snyder, C. L. \& Lewis, D. A. (1995). Axon terminals immunolabeled for dopamine or tyrosine hydroxylase synapse on GABA-immunoreactive dendrites in rat and monkey cortex. Journal of Comparative Neurology (in the press).

Smiley, J. F. \& Goldman-Rakic, P. S. (1993). Heterogeneous targets of dopamine synapses in monkey prefrontal cortex demonstrated by serial section electron microscopy: a laminar analysis using the silver-enhanced diaminobenzidine sulfide (SEDS) immunolabeling technique. Cerebral Cortex 3, 223-238.

T'so, D. Y. \& Gilbert, C. D. (1988). The organization of chromatic and spatial interactions in the primate striate cortex. Journal of Neuroscience 8, 1712-1727.

T'so, D. Y., Gilbert, C. D. \& Wiesel, T. N. (1986). Relationships between horizontal connections and functional architecture in cat striate cortex as revealed by cross-correlation analysis. Journal of Neuroscience 6, 1160-1170.

Wallace, M. N., Kitzes, L. M. \& Jones, E. G. (1991). Intrinsic interand intra-laminar connections and their relationship to the tonotopic map in cat primary auditory cortex. Experimental Brain Research 86, 527-544.

Weinberger, D. R. (1987). Implications of normal brain development for the pathogenesis of schizophrenia. Archives of General Psychiatry 44, 660-669.

Weinberger, D. R., Berman, K. F. \& Zec, R. F. (1986). Physiological dysfunction of dorsolateral prefrontal cortex in schizophrenia. 1 . Regional cerebral blood flow (rCBF) evidence. Archives of General Psychiatry 43, 114-125.

Weinberger, D. R., Berman, K. F. \& Illowsky, B. P. (1988). Physiological dysfunction of dorsolateral prefrontal cortex in schizophrenia: III. A new cohort and evidence for a monoaminergic mechanism. Archives of General Psychiatry 45, 609-615.

White, E. L. (1989). Cortical Circuits. Synaptic Organization of the Cerebral Cortex. Structure, Function and Theory (ed. A. Keller), pp. 5-223. Birkhauser: Boston, Basel, Berlin.

Yoshioka, T., Levitt, J. B. \& Lund, J.S. (1992). Intrinsic lattice connections of macaque monkey visual cortical area V4. Journal of Neuroscience 12, 2785-2802.

Zipursky, R. B., Lim, K. O., Sullivan, E. V., Brown, B. W. \& Pfefferbaum, A. (1993). Widespread cerebral gray matter volume deficits in schizophrenia. Archives of General Psychiatry 49, 195-205. 